\title{
Understanding attitudes to inequality
}

\author{
In the face of growing economic inequality, rebalancing the wealth gap at global and national levels is \\ key to alleviating health, educational and lifestyle inequalities - but could our respect for established \\ hierarchies hinder a move toward fairer distribution?
}

Economic inequality has become a serious concern for many in the political, public and academic spheres. The gap between rich and poor has been increasing for decades, to the extent that more than $50 \%$ of the world's wealth is now held by the top $1 \%$ of earners ${ }^{1}$. The geography of inequality can be mapped against poorer health standards, lesser educational attainment, higher crime rates ${ }^{2,3}$, greater unhappiness and lack of trust in one's fellow citizens ${ }^{4}$.

Research in psychology and economics provides a seeming paradox when compared with these grim statistics of growing inequality in wealth and income. Numerous laboratory-based studies have shown that people reject unfair payment distributions ${ }^{5,6}$. Participants even reject inequitable outcomes on behalf of unknown others in third-party scenarios. This work suggests that equality should be a powerful and desirable social norm. However, if this is the case, why is wealth inequality so tolerated in real life?

A paper published in this issue of Nature Human Behaviour by Xie et al. ${ }^{7}$ tackled one aspect of this question by asking whether people are willing to overturn an established wealth hierarchy to achieve equality. The authors constructed a type of third-party dictator game that they call the redistribution game. Participants were presented with a pair of unknown players who had received a random allocation of coins at the start of the game. Four scenarios were given in which coins were redistributed from the richer to the poorer player, which the participants could choose to support or reject. Three of the scenarios proposed redistributions of different size that did not change the wealth hierarchy. However, one of the scenarios resulted in a redistribution where the poorer and wealthier player changed positions in the wealth ranking. The experiment was cross-cultural, having been conducted with Chinese, Indian and American participants and also with adult members of a Tibetan herding community, which has low market integration. Across all groups, a more equal distribution was preferred, but there was a majority aversion to reversing the rank of the two players. An increase in the participants' socioeconomic status or holding more conservative political values also predicted higher likelihood of aversion to rank reversal.

A version of the redistribution game was adapted for children. Consistent with previous research ${ }^{5}$, it was found that 4-year-olds rejected unequal distributions. However, by the age of 6-7 years old, children began to also express aversion to overturning hierarchies. Although this study cannot reveal the developmental mechanism, it seems that respect for hierarchy may establish early as a social norm across human cultures and is one that contends with equality.

The apparent disparity between a norm for equality observed in lab experiments and the many-tiered structure of real life was the subject of a Perspective by Starmans et al. ${ }^{8}$ in our April issue. In it, the authors argue that what the majority of people desire is not absolute economic equality, but fairness. Starman and colleagues point to experiments where, when asked to distribute income across large populations, participants actually prefer unequal societies. They argue that this difference comes down to a lack of ecological validity in most small-group lab experiments where, without any situational context, an even distribution is always the fairest one. In experiments, however, where an element of merit is introduced, participants, even young children, prefer to support merit-based inequality. Adults and children also both accept random or gameof-chance allocations where impartiality is the fairest decision maker ${ }^{9,10}$, as was the case at the start of the redistribution game in the study by Xie et al. Starmans' argument intersects with the findings by Xie et al. Both suggest that there is an aversion to interfering with an earned or deserved higher income. In the fairness paradigm posited by Starman, people are more concerned about over- and undercompensation than absolute equality weighting their perception of equality against what is earned and holding equality of opportunity as the ideal.

The notion of a meritocratic preference will make intuitive sense to many. The 'American Dream', for example, is a widely held belief that upward social mobility is possible for any person that applies hard work and ingenuity. However, in many nations, and most particularly the United States ${ }^{11}$, social mobility has decreased. When asked to estimate the difference between high and low earners, the average person's perception is way off the mark, as is the gap between what people believe is a fair difference in income for higher earners and the actual ratio of executive to worker pay. It would seem, therefore, that the current level of inequality or economic unfairness in some countries is beyond what the majority of people may find acceptable. However, could social norms such as an aversion to rank reversal present a barrier to enacting greater economic equality?

Asking people for their assessments of abstract scenarios concerning economic equality across a nation is one way to understand the type of society that people want to inhabit. However, we should also consider the terms on which people live their lives. People are surely concerned about equality in access to public services, health care and education. Economic inequality has a strong influence on these, and so correcting it is a way to ensure equality of opportunity. Making the distinction between fairness and equality should not interfere with this goal, but studying the psychology of social norms may guide what interventions or policies will be socially acceptable and likely to take root.

\footnotetext{
References

1. Global Wealth Report 2016 (Credit Suisse, 2016); http://go.nature.com/2uCOxxl

2. Dorling, D. Inequality and the $1 \%$ (Verso Books, 2014).

3. Wilkinson, R. \& Pickett, K. The Spirit Level: Why More Equal Societies Almost Always Do Better (Penguin Books Ltd, 2009).

4. Oishi, S., Kesebir, S. \& Diener, E. Psychol. Sci. 22, 1095-1100 (2011)

5. Fehr, E., Bernhard, H. \& Rockenbach, B. Nature 454, 1079-1083 (2008).

6. Charness, G., Cobo-Reyes, R. \& Jimenez, N. J. Econ. Behav. Organ. 68, 18-28 (2008).

7. Xie, W. et al. Nat. Hum. Behav. 1, 0142 (2017).

8. Starmans, C. et al. Nat. Hum. Behav. 1, 0082 (2017).

9. Kimbrough, E. O., Sheremeta, R. M. \& Shields, T. W. J. Econ. Behav. Organ. 99, 96-108 (2014).

10. Shaw, A. \& Olson, K. J. Exp. Child Psychol. 119, 40-53 (2014).

11. Carr, M. \& Wiemers, E. The Decline in Lifetime Earnings Mobility in the U.S.: Evidence from Survey-Linked Administrative Data (Washington Center for Equitable Growth, 2016); http://go.nature.com/2tfcEyM
} 